\title{
Fatores associados à Depressão Pós-Parto em mulheres em situação de vulnerabilidade social
}

\author{
André Luiz Monezi Andrade ${ }^{1}$ \\ Leila Regina da Silva Teixeira ${ }^{2}$ \\ Caroline Carmo Zoner ${ }^{3}$ \\ Nathália Nunes Niro $^{3}$ \\ Adriana Scatena ${ }^{4}$ \\ Ricardo Abrantes do Amaral ${ }^{5}$
}

O presente trabalho teve por objetivo avaliar possíveis fatores associados com a depressão pós-parto (DPP), como o uso de substâncias e o suporte psicossocial em uma amostra de 102 mulheres em situação de vulnerabilidade social. O estudo foi realizado com puérperas que residiam provisoriamente no alojamento de um Hospital Maternidade público. Aproximadamente $20 \%$ das puérperas apresentaram critérios para a DPP além de um padrão de uso de álcool e maconha superior àquelas que não tinham o transtorno. Observou-se também associação entre violência, falta de suporte psicológico e apoio familiar com a DPP. Observou-se uma associação entre a DPP com outras comorbidades de modo que algumas variáveis estudadas possam estar envolvidas na etiologia e manutenção deste transtorno.

Descritores: Transtorno Depressivo; Transtornos Relacionados ao Uso de Substâncias; Período Pós-Parto; Vulnerabilidade Social.

\footnotetext{
${ }^{1}$ Pós-doutorando, Universidade Federal de São Paulo, São Paulo, SP, Brasil. Professor Doutor, Universidade Anhembi Morumbi, São Paulo, SP, Brasil.

${ }^{2}$ Aluna do curso de graduação em Enfermagem, Universidade Anhembi Morumbi, São Paulo, SP, Brasil.

${ }^{3}$ Médica.

${ }^{4}$ Mestranda, Universidade Federal de São Paulo, São Paulo, SP, Brasil.

${ }^{5} \mathrm{PhD}$, Professor Doutor, Universidade Anhembi Morumbi, São Paulo, SP, Brasil.
}

\author{
Endereço de correspondência: \\ André Luiz Monezi Andrade \\ Universidade Anhembi Morumbi \\ Rua Dr. Almeida Lima, 1134 \\ Bairro: Mooca. \\ CEP: 03164-000, São Paulo, SP, Brasil \\ E-mail: andremonezi@gmail.com
}




\title{
Factors associated with postpartum depression in social vulnerability women.
}

This study aimed to evaluate possible factors associated with postpartum depression syndrome (PDD), as the substance abuse and psychosocial support in a sample of 102 women in a social vulnerability condition. The study was conducted with mothers who were residents in specific hospital's lodging. Almost $20 \%$ of mothers showed symptoms to PDD as well as abusive alcohol and marijuana consumption compared with those who did not have PDD. Besides, it was observed an association between lack of psychological and familiar support with PDD. PDD it seems to be associated with other comorbidities so that some of these variables can be linked on the etiology and maintenance of PDD.

Descriptors: Depressive Disorder; Substance-Related Disorders; Postpartum Period; Social Vulnerability.

\section{Factores asociados con la depresión posparto en las mujeres en situación de vulnerabilidad social.}

\begin{abstract}
Este estudo tuvo como objectivo evaluar possibles factores associados con la Depresión Posparto (DPP) como el uso de substancias y apoio psicossocial em 102 mujeres em situación de vulnerabilidad social. El estúdio se realizó com las madres residentes transitórias em la vivienda de un Hospital Maternidad. Aproximadamente $20 \%$ de las madres presentaron criterios para el DPP, así como un de consumo de alcohol y marihuana superior a aquellas que no tienen el trastorno. También fue observada uma asociación entre la violencia, falta de apoyo psicológico y de la familia con el DPP. El DPP parece estar asociada con otras enfermedades concomitantes por lo que algunas variables pueden estar implicados en la etiología y el mantenimiento de este trastorno.
\end{abstract}

Descriptores: Transtorno Depressivo; Transtornos Relacionados con Sustancias; Periodo Posparto; Vulnerabilidad Social.

\section{Introdução}

A Depressão Pós-Parto (DPP) é um transtorno mental caracterizado por alterações persistentes de humor deprimido, além da perda de interesse e/ou prazer por atividades diárias, alterações das funções vegetativas (sono, libido, apetite etc.) e psicológicas ${ }^{(1)}$. Atualmente, estima-se cerca de 350 milhões de pessoas com depressão no mundo, e no caso da DPP sua prevalência é bastante variada, dependendo do país e da metodologia utilizada. Em uma revisão sistemática realizada por Norhayati e colaboradores ${ }^{(2)}$, foram identificados 203 artigos de 42 países e os autores observaram uma prevalência de DPP variando entre $1,9 \%$ a $82,1 \%$ nos países em desenvolvimento e entre $5,2 \%$ a $74 \%$ em países desenvolvidos. Estes dados foram baseados em instrumentos autoaplicados e, segundo os autores, observou-se uma menor prevalência de DPP a partir de entrevistas clínicas estruturadas (entre $0,1 \%$ a $26,3 \%$ ).

No Brasil, uma revisão sistemática avaliando 14 trabalhos identificou uma prevalência com aproximada entre 8 a $40 \%$, sendo que na maioria dos estudos, os pesquisadores utilizaram questionários 
autoaplicados $^{(3)}$. Em outro trabalho avaliando a prevalência de DPP a partir de uma entrevista clínica estruturada do DSM-IV, 7\% das mulheres da cidade de Recife-PE receberam o diagnóstico(4). Mais recentemente, Corrêa e colaboradores ${ }^{(5)}$ identificaram a prevalência de DPP em 19\% e 20,3\% das mulheres das regiões do Nordeste e do Estado do Amazonas, respectivamente.

A DPP é um transtorno mental que pode levar a graves consequências tanto nas mulheres, quanto nos bebês e pessoas de convívio próximo. Em relação às crianças cujas mães tiveram DPP, 17\% delas apresentaram atrasos de desenvolvimento psicomotor e maiores problemas de aprendizagem a partir de um estudo longitudinal(6). Além disso, mulheres com DPP podem apresentar maior dificuldade de interação com o bebê, dinâmica familiar, além de uma piora da qualidade de vida(7).

Em relação a sua etiologia, a DPP parece ter causas multifatoriais, especialmente a partir de fatores biológicos, psicológicos e socioeconômicos. Em relação aos primeiros, as evidências mais robustas concentramse em alterações hormonais, como a progesterona, estrógenos gonadais e prolactina( ${ }^{(8)}$ que alteram a modulação dos sistemas de neurotransmissão, especialmente os sistemas serotoninérgico e noradrenérgico. No caso de variáveis psicológicas, episódios prévios de depressão pode aumentar as chances de um episódio de DPP em 25 a $50 \%{ }^{(9)}$. Além disso, em mulheres que apresentaram algum Transtorno de Ansiedade, observou-se maior chance de comorbidades com a DPP(10). Em relação aos aspectos socioeconômicos, existe forte associação entre a DPP com a falta de suporte familiar e social(11), além de baixo nível socioeconômico(12), frequências de brigas com o parceiro(13) e abuso de substâncias.

Em relação a este último, Certain e colaboradores ${ }^{(14)}$ detectaram uma chance quase 4 vezes maior de DPP em mulheres com histórico de abuso de substâncias. Em outro estudo(15), 20\% das mulheres com DPP apresentaram uma história de abuso de substâncias em relação àquelas que não tinham DPP. Além disso, neste mesmo trabalho, os pesquisadores detectaram que aproximadamente $47 \%$ do grupo DPP e $17 \%$ do grupo controle relataram ter utilizado pelo menos uma substância ilícita durante a gravidez.

O presente trabalho, teve por objetivo avaliar a associação entre a DPP e o uso de substâncias em mulheres residentes em um alojamento provisório de uma Maternidade pública da Cidade de São Paulo. Além disso, pretendeu-se avaliar possíveis diferenças socioeconômicas entre mulheres com DPP em relação àquelas que não apresentavam o Transtorno. A hipótese principal deste estudo é a de que mulheres com DPP apresentariam maior probabilidade de consumo de substâncias e que apresentariam maior frequência de dificuldades socioeconômicas (violência familiar, falta de suporte social, renda etc.).

\section{Métodos}

\section{Amostra}

A amostra foi composta por 102 puérperas que residiam provisoriamente no alojamento conjunto de um Hospital Maternidade Escola da Cidade de São Paulo durante os primeiros dias após o parto. O alojamento conjunto funciona como um abrigo provisório tanto para gestantes quanto para puérperas que se encontram em situação de vulnerabilidade social (falta de casa, problemas familiares, e outras situações de amaça a integridade física e/ou emocional).

\section{Instrumentos}

Os instrumentos utilizados consistiram de um questionário sociodemográfico composto por 13 questões gerais (gênero, idade, etnia, estado civil, escolaridade, renda, se recebe ajuda psicológica, recebe ajuda de familiares e se já foi vítima de violência, número de gestações, filhos e abortos e modalidade do último parto) e desenvolvido pelos autores. Além disso, foram utilizados dois instrumentos padronizados;

1. Escala de Depressão Pós-Parto de Edimburg (EPDS): Este é um instrumento auto aplicado composto por 10 questões relacionados a sintomas específicos da DPP (humor deprimido ou disfórico, alterações do sono, alteração de apetite, perda de prazer, ideação suicida, diminuição de desempenho e ideias de culpa). As questões são pontuadas entre 0 a 3, com um máximo de 30 pontos, de acordo com a presença e intensidade dos sintomas. Embora existam diferentes procedimentos de classificação nesse instrumento, neste estudo as puérperas foram classificadas somente em dois grupos; Grupo Sem Risco (GSR) e Grupo Depressão Pós-Parto (GDPP) De acordo com a pontuação, baseando-se em trabalhos anteriores ${ }^{(16)}$, as entrevistadas eram classificadas como GDPP quando sua pontuação era maior do que 10 pontos na escala. As pontuações menores ou iguais a 10 foram classificadas como GSR. A amostra do presente estudo apresentou diferenças significantes entre os dois grupos 
$\left(F_{(1,100)}=191,3\right)$, assim como um tamanho de efeito aceitável (eta square; $n \rho^{2}=0,66$ ).

2. Alcohol, Smoking and Substance Involvement Screening Test (ASSIST): Este instrumento foi desenvolvido por pesquisadores da Organização Mundial da Saúde e tem por objetivo avaliar o consumo de substâncias nos últimos três meses. O instrumento é composto por 8 itens e deve ser aplicado e interpretado por profissional da saúde, pois possui pontuações específicas, dependendo do consumo das substâncias em questão. As propriedades psicométricas do instrumento foram validadas na população brasileira, com resultados considerados robustos.

\section{Procedimentos}

Após a apresentação da Carta de Aprovação do Comitê de Ética em Pesquisa ao Hospital e a autorização da Instituição, as puérperas foram convidadas a participarem deste estudo e aquelas que aceitaram agendaram uma entrevista com os pesquisadores para a apresentação formal do Termo de Consentimento e posteriormente a coleta de dados. Tanto o agendamento quanto a coleta foram realizados no Ambulatório Umbigo, a partir de uma ala específica do Hospital Maternidade Escola. Cada entrevista teve duração aproximada entre 20 a 30 minutos, e foi realizada por estudantes treinados para aplicação dos instrumentos do curso de Medicina ( $6^{\circ}$ ano) da Universidade Anhembi Morumbi.

Antes do início da coleta de dados, um procedimento piloto foi realizado com alguns alunos da Universidade sem caráter de pesquisa, mas somente para avaliar a clareza das perguntas e o fluxo da aplicação dos instrumentos. Estes dados não foram inseridos neste estudo e os ajustes necessários foram realizados para o posterior procedimento da coleta.

\section{Análise de Dados}

Os dados referentes às variáveis contínuas foram padronizados e transformados em Z score com o objetivo de se detectar possíveis padrões de respostas outliers que pudessem prejudicar a confiabilidade das análises. Assim, valores abaixo ou acima de 3 desvios padrão foram excluídos de análises específicas. Desta forma, das 102 púerperas que compuseram a amostra total, foram identificados e excluídos 28 resultados outliers, sendo 4 para a variável "idade, 3 para a "renda", 3 para "quantidade de gestações", 9 para "quantidade de abortos", e 9 para "quantidade de filhos".

Para a análise das variáveis categóricas utilizouse o teste do $X^{2}$ sendo que para as contínuas utilizou-se a Análise de Variância (ANOVA). Quando possível, optou-se pela apresentação não somente do nível de significância, mas também do valor dos Testes e do tamanho do efeito das análises, conforme recomendação da Associação Americana de Psicologia(17). Em relação ao tamanho do efeito, quando utilizada a ANOVA, utilizou-se o Teste Eta Square $\left(\eta \rho^{2}\right)$. As análises foram realizadas por meio do programa Statistica 16.0 (Statsoft Inc®).

\section{Ética}

O estudo foi submetido e aprovado pelo Comitê de Ética e Pesquisa da Universidade Anhembi Morumbi (CAAE 18748013.2.0000.5492), número 432.804.

\section{Resultados}

Do total das 102 puérperas, 21 (20,6\%) apresentaram uma pontuação acima de 10 no instrumento EPDS, sendo classificadas no grupo GDPP e as demais ( $\mathrm{N}=81$, $79,4 \%$ ) foram classificadas no grupo GSR. A Tabela 1 indica os dados sociodemográficos em relação aos grupos (GSR e GDPP).

Tabela 1 - Descrição dos dados sociodemográficos, nível de gravidade da Escala de Depressão Pós-Parto de Edimburg (EPDS). São Paulo, SP, Brasil (2015), (N=101).

\begin{tabular}{|c|c|c|c|c|c|c|}
\hline & $\begin{array}{c}\text { Sem Risco } \\
\qquad \mathbf{N}=81\end{array}$ & $\begin{array}{c}\text { Depressão } \\
\text { Pós Parto } \\
\text { N=21 }\end{array}$ & Teste & $p$ & $\begin{array}{l}\text { Tamanho } \\
\text { de efeito }\end{array}$ & $\begin{array}{c}\text { Poder } \\
\text { observado }\end{array}$ \\
\hline Idade & $26( \pm 6,3)$ & $25,6( \pm 6,1)$ & $0,07^{\text {(a) }}$ & $0,78^{(\mathrm{a})}$ & $0,00^{(b)}$ & $0,05^{(\mathrm{c})}$ \\
\hline Etnia & & & $4,37^{(\mathrm{d})}$ & $0,11^{(\mathrm{b})}$ & $0,2^{(\mathrm{e})}$ & \\
\hline Branca & $36(45 \%)$ & $7(33,3 \%)$ & & & & \\
\hline Parda & $33(41,7 \%)$ & $7(33,3 \%)$ & & & & \\
\hline Negra & $11(13,3 \%)$ & $7(33,3 \%)$ & & & & \\
\hline
\end{tabular}


Tabela 1 - continuação

\begin{tabular}{|c|c|c|c|c|c|c|}
\hline & $\begin{array}{c}\text { Sem Risco } \\
\quad N=81\end{array}$ & $\begin{array}{c}\text { Depressão } \\
\text { Pós Parto } \\
\text { N=21 }\end{array}$ & Teste & $p$ & $\begin{array}{l}\text { Tamanho } \\
\text { de efeito }\end{array}$ & $\begin{array}{c}\text { Poder } \\
\text { observado }\end{array}$ \\
\hline Renda bruta $^{(\mathfrak{)})}$ & $\begin{array}{c}508,8 \\
( \pm 221,82)\end{array}$ & $\begin{array}{c}412,96 \\
( \pm 223,15)\end{array}$ & $2,58^{(a)}$ & $0,12^{(a)}$ & $0,00^{(\mathrm{b})}$ & $0,35^{(\mathrm{c})}$ \\
\hline Escolaridade (em anos) & $9,1( \pm 2,95)$ & $8,1( \pm 2,93)$ & $1,98^{(a)}$ & $0,16^{(\mathrm{a})}$ & $0,02^{(b)}$ & $0,28^{(\mathrm{c})}$ \\
\hline Estado civil & & & $3,16^{(d)}$ & $0,07^{(b)}$ & $0,2^{(e)}$ & \\
\hline Solteira & $29(35,8 \%)$ & $12(57,1 \%)$ & & & & \\
\hline Casada & $52(64,2 \%)$ & $9(42,9 \%)$ & & & & \\
\hline Quantidade de filhos & $1,8( \pm 0,93)$ & $2,2( \pm 0,93)$ & $2,48^{(\mathrm{a})}$ & $0,11^{\text {(a) }}$ & $0,03^{(b)}$ & $0,36^{(\mathrm{c})}$ \\
\hline Quantidade de abortos & $0,34( \pm 0,6)$ & $0,33( \pm 0,61)$ & $0,00^{(a)}$ & $0,96^{(\mathrm{a})}$ & $0,00^{(b)}$ & \\
\hline
\end{tabular}

(a)Análise de Variância- ANOVA ${ }^{(b)}$ Teste Eta Square $\left(\eta \rho^{2}\right)^{(\mathrm{c})}$ No caso da ANOVA, foi inserido o poder observado das análises ${ }^{(\mathrm{d})}$ Teste de Qui-Quadrado ${ }^{(e)}$ Teste de Cramer ${ }^{(f)}$ Cotação média do Real em Julho de 2015; \$1dólar= R\$ 3,30 reais.

Em relação à idade, não foram observadas diferenças, ainda que o GSR tenha apresentado uma idade ligeiramente maios que o GDPP. Em relação à etnia, aproximadamente $45 \%$ das mulheres do GSR eram brancas, enquanto esta distribuição foi mais uniforme para o GDPP (33\% para cada grupo). Além disso, as mulheres do GSR tiveram uma renda média aproximadamente $\$ 91$ dólares a mais e um ano a mais de tempo médio de escolaridade em relação ao GDPP, embora sem diferenças significantes pela ANOVA para estas duas variáveis. Aproximadamente $65 \%$ das mulheres do GSR relataram serem casadas ou morarem com seus parceiros (união estável) em comparação ao GDPP (43\%), sendo que a quantidade de filhos neste último grupo foi ligeiramente superior (2,2 filhos) em comparação ao GSR (1,8 filhos). Por fim, em relação a quantidade de abortos relatadas pelas mulheres, os índices foram muito semelhantes entre os grupos GSR $(0,34)$ e $\operatorname{GDPP}(0,33)$.

A Tabela 2 indica diferenças entre os GSR e GDPP em relação às pontuações médias para as três substâncias detectadas na amostra (álcool, tabaco e maconha) nos últimos três meses, além de algumas características comportamentais das puérperas.

Tabela 2 - Descrição dos dados relacionados à pontuação do instrumento Alcohol, Smoking and Substance Involvement Screening Test (ASSIST), e comportamentais das entrevistadas a partir do nível de gravidade da Escala de Depressão Pós-Parto de Edimburg (EPDS). São Paulo, SP, Brasil (2015), (N=101).

\begin{tabular}{|c|c|c|c|c|c|c|}
\hline & $\begin{array}{c}\text { Sem Risco } \\
\quad N=81\end{array}$ & $\begin{array}{c}\text { Depressão } \\
\text { Pós Parto } \\
\text { N=21 }\end{array}$ & Teste & $p$ & $\begin{array}{c}\text { Tamanho de } \\
\text { efeito }\end{array}$ & $\begin{array}{c}\text { Poder } \\
\text { observado }\end{array}$ \\
\hline \multicolumn{7}{|l|}{ ASSIST } \\
\hline Tabaco & $2,62( \pm 6,53)$ & $4,55( \pm 6,76)$ & $1,3^{(\mathrm{a})}$ & $0,3^{(a)}$ & $0,01^{(b)}$ & $0,2^{(\mathrm{c})}$ \\
\hline Álcool & $1,02( \pm 2,97)$ & $2,85( \pm 3)$ & $6,3^{(a)}$ & $0,01^{\text {(a) }}$ & $0,06^{(\mathrm{b})}$ & $0,7^{(c)}$ \\
\hline Maconha & $0,07( \pm 0,66)$ & $0,45( \pm 0,67)$ & $5,2^{(a)}$ & $0,02^{(a)}$ & $0,05^{(b)}$ & $0,62^{(\mathrm{c})}$ \\
\hline Gestação Planejada & & & $0,1^{(d)}$ & $0,74^{(\mathrm{d})}$ & $0,1^{(d)}$ & \\
\hline Sim & $57(70,4 \%)$ & $14(66,7 \%)$ & & & & \\
\hline Não & $24(29,6 \%)$ & $7(33,3 \%)$ & & & & \\
\hline Pré natal efetivo & & & $2,2^{(\mathrm{d})}$ & $0,13^{(d)}$ & $0,14^{(\mathrm{e})}$ & \\
\hline Sim & $79(97,5 \%)$ & $19(90,5 \%)$ & & & & \\
\hline Não & $2(2,5 \%)$ & $2(9,5 \%)$ & & & & \\
\hline Já foi vítima de violência? & & & $7,2^{(\mathrm{d})}$ & $0,00^{(\mathrm{d})}$ & $0,26^{(\mathrm{e})}$ & \\
\hline Sim & $6(7,4 \%)$ & $6(28,5 \%)$ & & & & \\
\hline Não & $75(92,6 \%)$ & $15(71,5 \%)$ & & & & \\
\hline $\begin{array}{l}\text { Recebe suporte } \\
\text { psicológico? }\end{array}$ & & & $2,3^{(d)}$ & $0,13^{(\mathrm{d})}$ & $0,14^{(\mathrm{e})}$ & \\
\hline $\operatorname{Sim}$ & $9(11,1 \%)$ & $5(23,8 \%)$ & & & & \\
\hline Não & $72(88,9 \%)$ & $16(76,2 \%)$ & & & & \\
\hline
\end{tabular}


Tabela 2 - continuação

\begin{tabular}{|c|c|c|c|c|c|c|}
\hline & $\begin{array}{l}\text { Sem Risco } \\
\qquad N=81\end{array}$ & $\begin{array}{c}\text { Depressão } \\
\text { Pós Parto } \\
\mathrm{N}=\mathbf{2 1}\end{array}$ & Teste & $p$ & $\begin{array}{c}\text { Tamanho de } \\
\text { efeito }\end{array}$ & $\begin{array}{c}\text { Poder } \\
\text { observado }\end{array}$ \\
\hline $\begin{array}{l}\text { Recebe apoio de amigos, } \\
\text { Parceiros, familiares? }\end{array}$ & & & $15,8^{(d)}$ & $0,00^{\text {(d) }}$ & $0,4^{(e)}$ & \\
\hline Sim & $66(81,5 \%)$ & $8(38,1 \%)$ & & & & \\
\hline Não & $15(18,5 \%)$ & $13(61,9 \%)$ & & & & \\
\hline Nascimento do bebê? & & & $4,54^{(\mathrm{d})}$ & $0,1^{(d)}$ & $0,21^{(\mathrm{e})}$ & \\
\hline A termo & $67(82,7 \%)$ & $13(61,9 \%)$ & & & & \\
\hline Pré-termo & $10(12,4 \%)$ & $5(23,8 \%)$ & & & & \\
\hline Pós-termo & $4(4,9 \%)$ & $3(14,3 \%)$ & & & & \\
\hline Tipo de parto & & & $0,99^{(d)}$ & $0,6^{(d)}$ & $0,01^{(\mathrm{e})}$ & \\
\hline Normal & $43(53,1 \%)$ & $12(57,1 \%)$ & & & & \\
\hline Cesariana & $28(34,6 \%)$ & $8(38,1 \%)$ & & & & \\
\hline Fórceps & $10(12,3 \%)$ & $1(4,8 \%)$ & & & & \\
\hline
\end{tabular}
de Qui-Quadrado ${ }^{(\mathrm{e})}$ Teste de Cramer.

De forma geral, o uso de substâncias foi maior entre as mulheres do GDPP, entretanto a Análise de Variância detectou diferenças significantes para o uso de álcool $\mathrm{e}$ maconha (Teste de Sheffé, $p<0,05$ ). No caso do álcool, a pontuação de uso foi aproximadamente 2,5 vezes maior e 6,5 vezes maior para o uso de maconha no GDPP. Em relação ao fato de terem planejado suas gestações aproximadamente $70 \%$ das mulheres de ambos os grupos relataram que planejaram a gravidez e quanto ao de terem tido um cuidado Pré-natal efetivo, observouse uma resposta positiva para aproximadamente $97 \%$ do GSR e $90 \%$ do GDPP. Em relação ao fato de já terem sido vítimas de violência doméstica pelos seus parceiros, quase $30 \%$ das mulheres do GDPP afirmaram que sim, em comparação ao GSR $(7,5 \%)$. Entretanto, somente $23 \%$ das mulheres do GDPP recebem algum tipo de suporte psicológico. Em relação ao suporte de amigos e familiares no cuidado com a criança, enquanto mais de $80 \%$ das mulheres do GSR relataram receber alguma ajuda, observou-se que somente $38 \%$ das mulheres do GDPP afirmaram receber algum suporte. Em relação ao tipo de nascimentos, embora não tenham sido detectadas diferenças significantes, observouse o dobro de nascimentos pré-termo e o triplo de nascimento pós termo no GDPP.

A Tabela 3 indica as principais diferenças entre a pontuação bruta do EPDS, o consumo de substâncias e das variáveis comportamentais entre as puérperas que recebem (grupo SIM) e não recebem (grupo NÃO) apoio de familiares/amigos em relação ao recém-nascido.

Tabela 3 - Diferenças entre as puérperas que afirmaram receber (SIM) e não receber (NÃO) apoio de familiares e/ou amigos no cuidado à(s) criança(s) em relação à última gestação. São Paulo, SP, Brasil (2015), (N=101).

\begin{tabular}{|c|c|c|c|c|c|c|}
\hline & $\begin{array}{c}\text { SIM } \\
N=74\end{array}$ & $\begin{array}{l}\text { NÃO } \\
\mathrm{N}=28\end{array}$ & Teste & $p$ & Tamanho de efeito & Poder observado \\
\hline EPDS & 5,21 & 9,28 & $14,6^{(\mathrm{a})}$ & $0,00^{(a)}$ & $0,12^{(\mathrm{b})}$ & $0,96^{(\mathrm{c})}$ \\
\hline \multicolumn{7}{|l|}{ ASSIST } \\
\hline Tabaco & 2,64 & 4,08 & $0,85^{(\mathrm{a})}$ & $0,35^{(a)}$ & $0,00^{(\mathrm{b})}$ & $0,15^{(\mathrm{c})}$ \\
\hline Álcool & 0,66 & 3,33 & $18,71^{\text {(a) }}$ & $0,00^{(a)}$ & $0,16^{(\mathrm{b})}$ & $0,98^{(\mathrm{c})}$ \\
\hline Maconha & 0,08 & 0,37 & $3,65^{(\mathrm{a})}$ & $0,06^{(a)}$ & $0,03^{(\mathrm{b})}$ & $0,47^{(\mathrm{c})}$ \\
\hline Gestação Planejada & & & $2,86^{(\mathrm{d})}$ & $0,09^{(d)}$ & $0,16^{(\mathrm{d})}$ & \\
\hline Sim & $26(35,1 \%)$ & $23(82,1 \%)$ & & & & \\
\hline Não & $48(64,9 \%)$ & $5(17,9 \%)$ & & & & \\
\hline Pré natal efetivo & & & $4,72^{(\mathrm{d})}$ & $0,02^{(d)}$ & $0,21^{(\mathrm{e})}$ & \\
\hline Sim & $73(98,7 \%)$ & $25(89,3 \%)$ & & & & \\
\hline Não & $1(1,3 \%)$ & $3(10,7 \%)$ & & & & \\
\hline $\begin{array}{l}\text { Já foi vítima de } \\
\text { violência? }\end{array}$ & & & $6,51^{(\mathrm{d})}$ & $0,01^{(d)}$ & $0,24^{(\mathrm{e})}$ & \\
\hline Sim & $5(6,8 \%)$ & $7(25 \%)$ & & & & \\
\hline Não & $69(93,2 \%)$ & $21(75 \%)$ & & & & \\
\hline
\end{tabular}


Tabela 3 - continuação

\begin{tabular}{|c|c|c|c|c|c|c|}
\hline & $\begin{array}{c}\text { SIM } \\
N=74\end{array}$ & $\begin{array}{l}\text { NÃO } \\
\mathrm{N}=28\end{array}$ & Teste & $p$ & Tamanho de efeito & Poder observado \\
\hline $\begin{array}{l}\text { Recebe suporte } \\
\text { psicológico? }\end{array}$ & & & $7,18^{(\mathrm{d})}$ & $0,00^{(d)}$ & $0,27^{(\mathrm{e})}$ & \\
\hline Sim & $6(8,1 \%)$ & $8(28,6 \%)$ & & & & \\
\hline Não & $68(91,9 \%)$ & $20(71,4 \%)$ & & & & \\
\hline
\end{tabular}

(a)Análise de Variância- ANOVA ${ }^{(b)}$ Teste Eta Square $\left(n \rho^{2}\right)^{(\mathrm{c})}$ No caso da ANOVA, foi inserido o poder observado das análises ${ }^{(\mathrm{d})}$ Teste de Qui-Quadrado ${ }^{(e)}$ Teste de Cramer.

O grupo NÃO apresentou uma pontuação média para depressão pós-parto $80 \%$ maior em relação ao grupo SIM, além de um maior consumo de tabaco e maconha $(p<0,05)$ conforme detectado pelo instrumento ASSIST. Daquelas mulheres que recebem algum tipo de apoio, somente $35 \%$ planejaram sua gestação sendo que daqueles do grupo NÃO mais de $80 \%$ planejaram a gestação. Além disso, no grupo NÃO, $25 \%$ das mulheres relataram sofrer agressões em relação ao grupo SIM (7\%) e menos que $30 \%$ das mulheres do Grupo NÃO relataram receber algum suporte psicológico.

\section{Discussão}

Dentre os principais achados deste estudo, aproximadamente $20 \%$ das puérperas apresentavam critérios para a DPP sendo que estas mulheres apresentaram uma frequência maior no uso de substâncias em comparação com aquelas que não possuíam o transtorno. Além disso, observou-se associação entre violência, falta de suporte psicológico e apoio familiar com a DPP em comparação com aquelas que não possuíam o transtorno. As puérperas com DPP relataram que não possuíam este suporte familiar, apresentaram maiores pontuações para DPP e para uso de álcool, além de frequência de relatos de violência e falta de um suporte psicológico.

Em relação à prevalência, os dados encontrados neste estudo são próximos de outros trabalhos brasileiros que também utilizaram o mesmo instrumento, com prevalência aproximada de 20 a 25\% ${ }^{(03-05)}$. Em relação ao uso de substâncias, embora tenha sido detectado maior consumo entre as mulheres com DPP, especialmente para álcool e maconha, o efeito desta relação precisa ser melhor estudado pois ainda não é claro na literatura se mulheres com DPP podem aumentar o consumo de substâncias como forma de automedicação, ou o uso prolongado de substâncias pode desencadear processos neuroadaptativos que alteram os sistemas de neurotransmissão, especialmente as monoaminas, ou ainda se a associação entre DPP e o uso de substâncias está associado a aspectos sociais. Em relação ao primeiro caso, a insônia é um sintoma muito comum na DPP e o uso de álcool e maconha, devido a alguns efeitos relaxantes, pode ser utilizado como um indutor de sono (automedicação). Em relação ao uso crônico de sustâncias, existem evidências para o desenvolvimento de transtornos depressivos, especialmente a partir do uso crônico de drogas estimulantes (anfetaminas, metanfetaminas, cocaína, crack etc.) a partir de um efeito de auto regulação dos receptores monoaminérgicos e redução da produção de monoaminas. Em um estudo recente avaliando mais de 34.000 mulheres francesas ${ }^{(18)}$, os pesquisadores observaram que aquelas com histórico de abuso de substâncias, apresentaram uma chance quase três vezes maior de serem diagnosticadas com DPP.

Observou-se uma associação entre o GDPP com a falta de suporte familiar e a violência familiar e/ou a partir de um parceiro ${ }^{(19-2)}$. Estes dados estão alinhados com outros achados da literatura, em que estas situações experenciadas pelas puérperas podem atuar como fatores de risco para a etiologia do transtorno. Em um estudo avaliando puérperas que imigraram para Taiwan(11), os pesquisadores avaliaram de forma longitudinal o efeito de três modelos de suporte social (emocional, instrumental e informacional) às mulheres com DPP. Ao longo do tempo, embora todos tivessem um peso nos modelos de regressão, os suportes emocional e instrumental apresentaram uma correlação inversamente proporcional com a DPP, de modo que quanto maior o apoio, menor os índices de DPP. Em um estudo realizado com 38 puérperas chinesas ${ }^{(21)}$, dentre os maiores estressores que poderiam agravar a DPP, elas citaram a falta de apoio e as brigas conjugais, que podem acabar por afastar ainda mais potenciais laços emocionais e afetivos com pessoas que poderiam apoiá-las. Desta forma, o suporte familiar parece ser uma das variáveis mais fortemente associadas com a DPP, e no caso deste estudo quase $60 \%$ das pacientes $\mathrm{cm}$ DPP não recebiam suporte familiar. Além disso, no caso do suporte especializado, aproximadamente $24 \%$ do GDPP também recebiam algum apoio, o que pode aumentar a vulnerabilidade destas mulheres e por consequência a gravidade do transtorno. 


\section{Conclusões}

Em suma, neste trabalho observou-se uma relação entre a DPP e o uso de maconha e álcool. Em relação àquelas mulheres que não receberam suporte social, elas apresentaram maiores pontuações para DPP na escala EPDS, além de maior consumo de álcool em comparação com aquelas que recebem algum tipo de suporte. Essas associações podem auxiliar equipes de obstetrícia e de seguimento pré-natal no sentido de prevenir a DPP ou ainda planejar suporte para gestantes com o perfil observado. O presente estudo possui limitações que devem ser levadas em consideração, como uma amostra relativamente pequena para realizar análises estatísticas mais robustas, permitindo avaliar possíveis associações com outras variáveis. Além disso, o ASSIST é um instrumento que permite detectar o consumo de substâncias nos últimos três meses, limitando a compreensão dos pesquisadores acerca de uma possível associação entre um histórico de uso de substâncias e a DPP. Outro ponto a ser ressalvado, é que muitos pesquisadores utilizam testes de auto aplicação, como a EPDS, entretanto, um diagnóstico mais confiável só é possível por meio de entrevista clínica estruturada com um especialista. Por fim, em alguns estudos, os modelos de suporte psicossocial são categorizados de maneira específica e, neste trabalho, as perguntas referentes a este item foram inseridas de maneira não categorizada (suporte emocional, instrumental e informacional).

\section{Referências}

1. Brummelte S, Galea LA. Postpartum depression: Etiology, treatment and consequences for maternal care. Horm Behav. 2016;77:153-66.

2. Norhayati MN, Hazlina NH, Asrenee AR, Emilin WM. Magnitude and risk factors for postpartum symptoms: a literature review. J Affect Disord. 2015;175:34-52.

3. Lobato G, Moraes CL, Reichenheim ME. Magnitude da depressão pós-parto no Brasil: uma revisão sistemática. Rev. Bras. Saude Mater. 2011;11(4):369-79.

4. Cantilino A, Zambaldi CF, Albuquerque TL, Paes JA, Montenegro ACP, Sougey EB. Postpartum depression in Recife - Brazil: prevalence and association with bio-sociodemographic factors. J. Bras. Psiquiatr. 2010;59(1):1-9.

5. Correa H, Castro ECT, Santos W, Romano-Silva MA. Postpartum depression symptoms among Amazonian and Northeast Brazilian women. JAffect Disord. 2016; 204:214-8. 6. McDonald S, Kehler H, Bayrampour H, Fraser-Lee $\mathrm{N}$, Tough S. Risk and protective factors in early child development: Results from the All Our Babies (AOB) pregnancy cohort. Res Dev Disabil. 2016;58:20-30.
7. Bodhare TN, Sethi P, Bele SD, Gayatri D, Vivekanand A. Postnatal quality of life, depressive symptoms, and social support among women in southern India. Women Health. 2015;55(3):353-65.

8. Gammie SC, Driessen TM, Zhao C, Saul MC, Eisinger $B E$. Genetic and neuroendocrine regulation of the postpartum brain. Front Neuroendocrinol. 2016;42:1-17. 9. World Health Organization (WHO). Mental health aspects of women's reproductive health. A global review of the literature. World Health Organization 2009.

10. Champagne AL, Brunault P, Huguet G, Suzanne I, Senon JL, Body G, et al. Personality disorders, but not cancer severity or treatment type, are risk factors for later generalised anxiety disorder and major depressive disorder in non metastatic breast cancer patients. Psychiatry Res. 2016; 236:64-70.

11. Chen HH, Hwang FM, Lin LJ, Han KC, Lin CL, Chien LY. Depression and Social Support Trajectories During 1 Year Postpartum Among Marriage-Based Immigrant Mothers in Taiwan. Arch Psychiatr Nurs. 2016;30(3):350-5. 12. Grote NK, Katon WJ, Russo JE, Lohr MJ, Curran M, Galvin E, et al. Collaborative Care for Perinatal Depression in Socioeconomically Disadvantaged Women: A Randomized Trial. Depress Anxiety. 2015;32(11):821-34.

13. Estefan LF, Coulter ML, VandeWeerd C. Depression in Women Who Have Left Violent Relationships: The Unique Impact of Frequent Emotional Abuse. Violence Against Women. 2016;22 (11):1397-413.

14. Certain HE, Mueller M, Jagodzinski T, Fleming M. Domestic abuse during the previous year in a sample of postpartum women. J Obstet Gynecol Neonatal Nurs. 2008;37(1): 35-41.

15. Bryan TL, Georgiopoulos AM, Harms RW, Huxsahl JE, Larson DR, Yawn BP. Incidence of postpartum depression in Olmsted County, Minnesota. A population-based, retrospective study. J Reprod Med. 1999;44 (4): 51-8.

16. Malloy-Diniz LF, Schlottfeldt CGMF, Figueira $P$, Neves FS, Corrêa H. Escala de Depressão Pós-Parto de Edimburg: análise fatorial e desenvolvimento de uma versão de seis itens. Rev Bras Psiquiatr. 2010;32:316-8. 17. Cumming G, Fidler F, Kalinowski P, Lai J. The statistical recommendations of the American Psychological Association Publication Manual: Effect sizes, confidence intervals, and meta-analysis. Australian Journal of Psychology. 2012;64(3):138-46.

18. Tebeka S, Le Strat Y, Dubertret C. Developmental trajectories of pregnant and postpartum depression in an epidemiologic survey. J Affect Disord. 2016;203:62-8. 19. Kita S, Haruna M, Matsuzaki M, Kamibeppu K. Associations between intimate partner violence (IPV) during pregnancy, mother-to-infant bonding failure, and postnatal depressive symptoms. Arch Womens Ment Health. 2016;19(4):623-34. 
20. Kothari CL, Liepman MR, Shama Tareen R, Florian P, Charoth RM, Haas SS, et al. Intimate Partner Violence Associated with Postpartum Depression, Regardless of Socioeconomic Status. J Affect Disord. 2016;20(6):1237-46.

21. Tang L, Zhu R, Zhang X. Postpartum Depression and Social Support in China: A Cultural Perspective. J Health Commun. 2016;21(9):1055-61. 